\title{
Valor diagnóstico y pronóstico de la determinación de tripsinógeno-2 urinario en pacientes con pancreatitis aguda
}

\section{Usefulness of determining urinary trypsinogen-2 in diagnosis and prognosis of patients with acute pancreatitis}

\author{
J.A. Díaz Peromingo ${ }^{1}$, A. Albán ${ }^{2}$, P. Pesqueira ${ }^{1}$, S Molinos ${ }^{1}$, M.C. Gayol ${ }^{1}$
}

\section{RESUMEN}

Fundamento. Estudiar el papel del tripsinógeno-2 urinario en el diagnóstico y pronóstico inicial de pacientes con pancreatitis aguda (PA) así como su relación con la estancia media hospitalaria y la mortalidad.

Método. Se incluyeron 42 pacientes diagnosticados de PA a los que se realizó hematimetría, bioquímica sanguínea, amilasuria y tripsinógeno-2 urinario. Se estableció un punto de corte de $50 \mu \mathrm{g} / \mathrm{L}$ y una segunda dilución a $2.000 \mu \mathrm{g} / \mathrm{L}$. Otras variables incluidas fueron etiología, estancia media hospitalaria, traslado a UCI y fallecimiento.

Resultados. De los 42 pacientes, 29 (69\%) eran hombres y 13 (31\%) mujeres; edad media 61 años. La etiología más frecuente era la biliar, seguida del alcohol. Estancia media: 8,38 días. Traslado a UCI en $4(9,5 \%)$ pacientes. Dos de los pacientes trasladados a UCI y otro que no requirió traslado fallecieron $(7,14 \%)$. La amilasa sérica fue elevada en $33(78,57 \%)$ pacientes y la lipasa en $36(85,71 \%)$. El tripsinógeno urinario fue positivo en 34 pacientes (80,95\%). Se evidenció asociación entre tripsinógeno- 2 urinario y edad $(\mathrm{p}=0,016 ; \mathrm{r}=0,893)$, glucemia $(\mathrm{p}=0,005, \mathrm{r}=0,901)$, amilasa plasmática $(\mathrm{p}=0,029$; $r=0,852)$, lipasa $(p=0,022 ; r=0,809)$ e hipoxemia $(p=0,001$; $\mathrm{r}=0,962)$. En cuanto a la estancia media, se observó asociación estadística con edad $(\mathrm{p}=0,046 ; \mathrm{r}=0,784)$ y acidosis metabólica ( $\mathrm{p}=0,016 ; \mathrm{r}=0,839)$. La mortalidad se asoció a hipocalcemia $(\mathrm{p}=0,008 ; \mathrm{r}=0,899)$ y acidosis metabólica ( $\mathrm{p}=0,032 ; \mathrm{r}=0,814)$.

Conclusión. La determinación del tripsinógeno-2 urinario en pacientes con PA es un test útil y rápido. Los pacientes mayores de 65 años, con hipoxemia, acidosis metabólica e hipocalcemia tienen tendencia a presentar una estancia media hospitalaria prolongada y mayor mortalidad.

Palabras clave. Tripsinógeno-2 urinario. Pancreatitis aguda. Estancia media. Mortalidad.

\section{An. Sist. Sanit. Navar. 2009; 32 (3): 343-350}

1. Servicio de Medicina Interna. Hospital de Barbanza. Riveira. A Coruña.

2. Laboratorio. Hospital de Barbanza. Riveria. A Coruña.

Este trabajo ha sido parcialmente subvencionado por una beca de ayuda a la investigación de la Fundación Mutua Universal.

Recepción: 10 de marzo de 2009

Aceptación provisional: 1 de junio de 2009

Aceptación definitiva: 1 de septiembre de 2009

\begin{abstract}
Background. To study the role of urinary trypsinogen-2 in diagnosing and early prognosis of patients with acute pancreatitis (AP) and the relationship to length of hospital stay and mortality.
\end{abstract}

Methods. Forty-two patients were included in the study. In all cases, blood cell count, serum chemistry, urine amylase and urine trypsinogen-2 were measured. A cut-off of $50 \mu \mathrm{g} / \mathrm{L}$ was established and, when positive, a second dilution was made $(2000 \mu \mathrm{g} / \mathrm{L})$. Other variables included were etiology, mean length of hospital stay, transfer to an ICU and death.

Results. Out of the 42 patients, 29 (69\%) were men and $13(31 \%)$ women. Average age was 61 years. The most frequent cause was biliary, followed by alcohol. Mean hospital stay was 8.38 days. Transferred to an ICU: 4 (9.5\%) patients. Two of them and a third, who had not been transferred, died (7.14\%). High serum amylase was found in $33(78.57 \%)$ patients and high lipase in 36 (85.71\%). Urinary trypsinogen-2 was positive in 34 patients (80.95\%). Statistical association between urinary trypsinogen-2 and age ( $\mathrm{p}=0.016 ; \mathrm{r}=0.893)$, glucose $(\mathrm{p}=0.005$; $r=0.901)$, serum amylase $(p=0.029 ; r=0.852)$, lipase $(\mathrm{p}=0.022 ; \mathrm{r}=0.809)$ and hypoxemia $(\mathrm{p}=0.001 ; \mathrm{r}=0.962)$ was found. Regarding hospital stay, there was statistical association with age $(\mathrm{p}=0.046 ; \mathrm{r}=0.784)$ and metabolic acidosis $(\mathrm{p}=0.016 ; \mathrm{r}=0.839)$. With respect to mortality there was statistical association with hypocalcemia $(\mathrm{p}=0.008$; $\mathrm{r}=0.899)$ and metabolic acidosis ( $\mathrm{p}=0.032 ; \mathrm{r}=0.814)$.

Conclusion. Testing urinary trypsiongen-2 in patients with AP is rapid and useful. Patients over the age of 65 with hypoxia, metabolic acidosis and hypocalcemia tend to present a prolonged average hospital stay and higher mortality.

Key words. Urinary trypsinogen-2. Acute pancreatitis. Length of hospital stay. Mortality.

\section{Correspondencia}

José Antonio Díaz Peromingo

Doutor Teixeiro, $29-5^{\circ}$

15701. Santiago de Compostela (A Coruña)

Tfno. 609720996.

E-mail: jadiazperomingo@hotmail.com 


\section{INTRODUCCIÓN}

Se ha descrito que la incidencia de pancreatitis aguda (PA) en países occidentales puede oscilar entre 100-250 casos por millón de habitantes y año ${ }^{1}$. Esta enfermedad afecta de forma similar a hombres y mujeres en términos generales, si bien esto varía si se tiene en cuenta las distintas etiologías. En este sentido, la presencia de cálculos y barro biliares son más frecuentes en el sexo femenino mientras que la etiología alcohólica lo es en el masculino. Otras causas de PA, además de las ya citadas, incluyen las iatrogénicas (colangiopancreatografía retrógrada endoscópica [ERCP en sus siglas anglosajonas] y fármacos [fundamentalmente azatioprina, furosemida y salicilatos]), hipertrigliceridemia, hipercalcemia, hipotermia, neoplasias pancreáticas, infecciones virales o PA de origen hereditario. En un $80 \%$ de casos se trata de una afección leve, pero existe otro $20 \%$ de pacientes en los que la enfermedad puede ser grave y conllevar una importante morbimortalidad que puede llegar al 10\% cuando aparece el denominado síndrome de respuesta inflamatoria sistémi$\mathrm{ca}^{2}$. En este síndrome se produce una importante liberación de citocinas y aparición de infección y necrosis pancreática con la posibilidad de que se generen colecciones líquidas, abscesos o pseudoquistes ${ }^{3}$.

En el diagnóstico, además de la presentación clínica que puede ser variable, se requiere la confirmación mediante pruebas bioquímicas y de imagen. En nuestro medio las pruebas que se realizan de forma habitual son la determinación de amilasa y lipasa séricas y la amilasuria, si bien esta última es una técnica que se está quedando obsoleta. La importancia de contar con técnicas cada vez más sensibles y específicas que ayuden al clínico en el diagnóstico y en la evaluación pronóstica de los pacientes con esta entidad nosológica se antoja de gran importancia, sobre todo si se consiguen test rápidos que permitan hacer el diagnóstico y ayudar en la evaluación pronóstica a corto plazo ${ }^{4}$.

Con respecto al pronóstico se han estudiado también aproximaciones terapéuticas como el uso del ácido ursodeoxicólico previo a la colecistectomía en pacientes con pancreatitis aguda biliar con resultados desiguales desaconsejándose su uso de forma rutinaria ${ }^{5}$.

En el presente trabajo se pretende evaluar el papel de la determinación del tripsinógeno-2 urinario, un test de rápida realización, en el diagnóstico inicial y en la valoración pronóstica a corto plazo de pacientes con PA.

\section{MATERIAL Y MÉTODOS}

\section{Pacientes}

Se incluyeron en el estudio 42 pacientes diagnosticados de PA en base a criterios clínico-biológicos y de imagen. Para el diagnóstico se exigía sintomatología clínica compatible y elevación de las cifras de amilasa y/o lipasa séricas de al menos tres veces el nivel máximo considerado como normal en el laboratorio del hospital. Además se requirió evidencia de inflamación pancreática bien por estudios de imagen (ecografía abdominal y/o TAC abdominal) o demostrada en el momento de la cirugía en aquellos casos que la requirieron.

\section{Determinaciones analíticas}

A todos los pacientes se les realizó determinación de hemograma sanguíneo con recuento celular, gasometría arterial basal, bioquímica sérica con determinación de transaminasas, glucosa, fosfatasa alcalina, bilirrubina total y directa, urea, creatinina, calcio, iones, LDH, perfil lipídico, albúmina, amilasa y lipasa. El punto de corte tomado para la amilasa fue de $300 \mathrm{U} / \mathrm{L}$ y para la lipasa $600 \mathrm{U} / \mathrm{L}$ que son los comúnmente aceptados en la literatura en el diagnóstico de pancreatitis aguda ${ }^{6,7}$. Se determinó asimismo amilasa en orina y positividad para el tripsinógeno-2 urinario en el momento del diagnóstico. Las determinaciones de tripsinógeno-2 urinario se realizaron utilizando el Actim Pancreatitis test strip (Medix Biochemi$c a$, Finlandia) que es una prueba de inmunocromatografía. En este test se sumerge la tira reactiva en una muestra de orina del pa- 
ciente y el tripsinógeno-2 está unido a partículas de látex azules que están marcadas con anticuerpos monoclonales y que migran a través de una membrana de nitrocelulosa con una zona que contiene otro anticuerpo específico para otro epítopo del tripsinógeno-2. Se establece un punto de corte de 50 $\mu \mathrm{g} / \mathrm{L}$ a partir del cual se produce una línea azul en esta zona. El test se considera positivo si esta banda azul se desarrolla en los primeros 5 minutos de exposición. En las muestras consideradas positivas se realizó una segunda dilución considerando como nueva positividad la presencia de la banda azul a una concentración superior a 2.000 $\mu \mathrm{g} / \mathrm{L}$ para intentar valorar a los enfermos con posible enfermedad más grave. Todas las determinaciones analíticas se realizaron en las primeras 24 horas de hospitalización. Otras variables incluidas en el estudio fueron la etiología de la pancreatitis, la estancia media hospitalaria, el traslado a UCI y el fallecimiento de los pacientes.

\section{Análisis estadístico}

Para las variables cuantitativas se usó estadística descriptiva analizando media, desviación típica e intervalo. Las variables cualitativas se recogieron de forma dicotómica con excepción de la etiología de la pancreatitis aguda y en ellas se realizó un análisis descriptivo de frecuencias. La correlación entre las distintas variables analizadas se estudió mediante el coeficiente de correlación de Pearson cuando se trataba de variables continuas y el de Spearman para estudiar asociaciones entre variables continuas y no continuas tomando como positivas aquellas correlaciones con una significación (valor "p») menor de 0,05 . Se realizó un análisis de regresión lineal múltiple para las distintas variables ajustando por edad.

\section{RESULTADOS}

De los 42 pacientes incluidos, 29 (69\%) eran hombres y 13 (31\%) mujeres. La edad media fue de 61 (intervalo: 18-95) años. Los resultados de las variables analíticas estudiadas, expresados como media \pm desviación estándar se muestran en la tabla 1. La etiología más frecuente en nuestra serie fue la biliar seguida del alcohol y de la idiomática (Fig. 1). La estancia media fue de 8,38 (intervalo: 1-44) días, presentando 9 (21,4\%) pacientes una estancia mayor de 10 días. En cuanto al traslado a UCI, 4 $(9,5 \%)$ pacientes necesitaron ser trasladados. Dos de los pacientes trasladados a UCI y otro que no requirió traslado fallecieron (7,14\%). La determinación de amilasa sérica fue positiva en $33(78,57 \%)$ pacientes y la de lipasa en $36(85,71 \%)$. El tripsinógeno urinario fue positivo en 34 pacientes $(80,95 \%)$ y negativo en 8 (19,05\%). De los 34 pacientes con el test positivo, $12(35,29 \%)$ presentaron positividad a una dilución mayor de $2.000 \mu \mathrm{g} / \mathrm{L}$. En cuanto al análisis de correlación entre variables, se evidenció asociación positiva entre el tripsinógeno-2 urinario y edad $(\mathrm{p}=0,016 ; \quad \mathrm{r}=0,893)$, glucemia $(\mathrm{p}=0,005$; $\mathrm{r}=0,901)$, amilasa plasmática $(\mathrm{p}=0,029$; $r=0,852)$, lipasa $(p=0,022 ; r=0,809)$ e hipoxemia $(p=0,001 ; r=0,962)$. En particular esta asociación se hacía más acentuada para un nivel de glucemia mayor de 126 $(\mathrm{p}=0,001 ; \mathrm{r}=0,963)$. En cuanto a la estancia media, se observó correlación con la edad $(\mathrm{p}=0,046 ; \mathrm{r}=0,784)$ y la presencia de acidosis metabólica ( $\mathrm{p}=0,016 ; \mathrm{r}=0,839)$. La mortalidad se asoció con la presencia de hipocalcemia ( $\mathrm{p}=0,008 ; \mathrm{r}=0,899)$ y acidosis metabólica $(\mathrm{p}=0,032 ; \mathrm{r}=0,814)$ (Tabla 2). El análisis de regresión lineal múltiple ajustando por edad mostró que existía relación con la estancia media [coeficiente de regresión 0.27 ; IC $95 \%(0,18 ; 0,34)$; $\mathrm{p}=0,004]$ pero no con las otras variables. En la tabla 3 se muestran los resultados medios de amilasa, lipasa y negatividad del tripsinógeno-2 urinario de los pacientes en los que alguna de estas determinaciones ha sido normal o, en el caso del tripsinógeno, negativa. 
Tabla 1. Resultados analíticos de las variables analizadas.

\begin{tabular}{|c|c|}
\hline Variable & Valor \\
\hline \multicolumn{2}{|l|}{ Bioquímica sanguínea } \\
\hline Glucosa & $147,33 \pm 57,39(70-110) \mathrm{mg} / \mathrm{dL}$ \\
\hline Urea & $44,78 \pm 25,28(15-39) \mathrm{mg} / \mathrm{dL}$ \\
\hline Creatinina & $1,04 \pm 0,29(0,6-1,3) \mathrm{mg} / \mathrm{dL}$ \\
\hline GOT & $163,86 \pm 242,41(15-37) \mathrm{U} / \mathrm{L}$ \\
\hline GPT & $183,17 \pm 214,97(30-65) \mathrm{U} / \mathrm{L}$ \\
\hline Fosfatasa alcalina & $289,14 \pm 188,47(40-190) \mathrm{U} / \mathrm{L}$ \\
\hline LDH & $353,94 \pm 142,5(130-500) \mathrm{U} / \mathrm{L}$ \\
\hline Bilirrubina total & $2,45 \pm 2,91(0-1,3) \mathrm{mg} / \mathrm{dL}$ \\
\hline Bilirrubina directa & $1,66 \pm 2,81(0-0,4) \mathrm{mg} / \mathrm{dL}$ \\
\hline Sodio & $139,28 \pm 3,43(137-148) \mathrm{mEq} / \mathrm{L}$ \\
\hline Potasio & $4,12 \pm 0,56(3,6-5,2) \mathrm{mEq} / \mathrm{L}$ \\
\hline Calcio & $8,94 \pm 1,15(8,8-10,5) \mathrm{mg} / \mathrm{dL}$ \\
\hline Amilasa & $713,88 \pm 877,90(25-115) \mathrm{mg} / \mathrm{dL}$ \\
\hline Lipasa & $5476,76 \pm 5811,06(114-286) \mathrm{mg} / \mathrm{dL}$ \\
\hline Albúmina & $3,70 \pm 0,84(3,5-5,5) \mathrm{mg} / \mathrm{dL}$ \\
\hline Colesterol total & $152,47 \pm 38,61(<200) \mathrm{mg} / \mathrm{dL}$ \\
\hline Colesterol LDL & $100,73 \pm 31,71(<130) \mathrm{mg} / \mathrm{dL}$ \\
\hline Colesterol HDL & $36,97 \pm 11,21(>35) \mathrm{mg} / \mathrm{dL}$ \\
\hline Triglicéridos & $116,02 \pm 47,32(<150) \mathrm{mg} / \mathrm{dL}$ \\
\hline \multicolumn{2}{|l|}{ Bioquímica urinaria } \\
\hline Amilasa & $1883,23 \pm 2905,74(30-200) \mathrm{UI} / \mathrm{L}$ \\
\hline \multicolumn{2}{|l|}{ Hemograma } \\
\hline Leucocitos & $10188,1 \pm 4128,26(4000-10000) / \mathrm{mm}^{3}$ \\
\hline Hemoglobina & $13,44 \pm 2,31(12-18) \mathrm{g} / \mathrm{dL}$ \\
\hline Hematocrito & $39,18 \pm 6,38(37-53) \%$ \\
\hline \multicolumn{2}{|l|}{ Gasometría arterial } \\
\hline $\mathrm{pH}$ & $7,40 \pm 0,48(7,38-7,44)$ \\
\hline pO2 & $74,77 \pm 11,73(80-100) \mathrm{mmHg}$ \\
\hline Bicarbonato & $25,33 \pm 3,26(23-28) \mathrm{mEq} / \mathrm{L}$ \\
\hline
\end{tabular}

Tabla 2. Factores asociados a positividad del tripsinógeno urinario, aumento de la estancia media y mortalidad.

\begin{tabular}{lll}
\hline \multicolumn{1}{c}{ Tripsinógeno } & Estancia media & Mortalidad \\
\hline Edad & Edad & Hipocalcemia \\
Glucosa plasmática & Acidosis metabólica & Acidosis metabólica \\
Amilasa plasmática & & \\
Lipasa & \\
pO2 menor de $60 \mathrm{mmHg}$ & \\
\hline $\begin{array}{l}\text { El factor edad hace referencia a pacientes mayores de } 65 \text { años. Glucosa, amilasa y lipasa hacen referencia a valores superiores a los } \\
\text { puntos de corte de dichas variables. }\end{array}$
\end{tabular}


Tabla 3. Valores medios de amilasa, lipasa y negatividad del tripsinógeno-2 urinario en los pacientes en los que alguna de dichas determinaciones es normal o negativa.

\begin{tabular}{lcccc}
\hline Variables con valores normales & Amilasa (media) & Lipasa (media) & $\begin{array}{c}\text { Tripsinógeno negativo } \\
\text { (no de pacientes) }\end{array}$ & N \\
\hline AMILASA (<300 U/L) & & $3.064,72 \pm 2.948,84$ & 5 & 9 \\
LIPASA (<600 U/L) & $95,83 \pm 69,41$ & & 4 & 6 \\
TRIPSINÓGENO & $380,50 \pm 412,25$ & $2.421,41 \pm 3.849,62$ & 8 \\
\hline
\end{tabular}

Los valores se expresan como media \pm desviación estándar.

$\mathrm{N}$ : número total de pacientes.

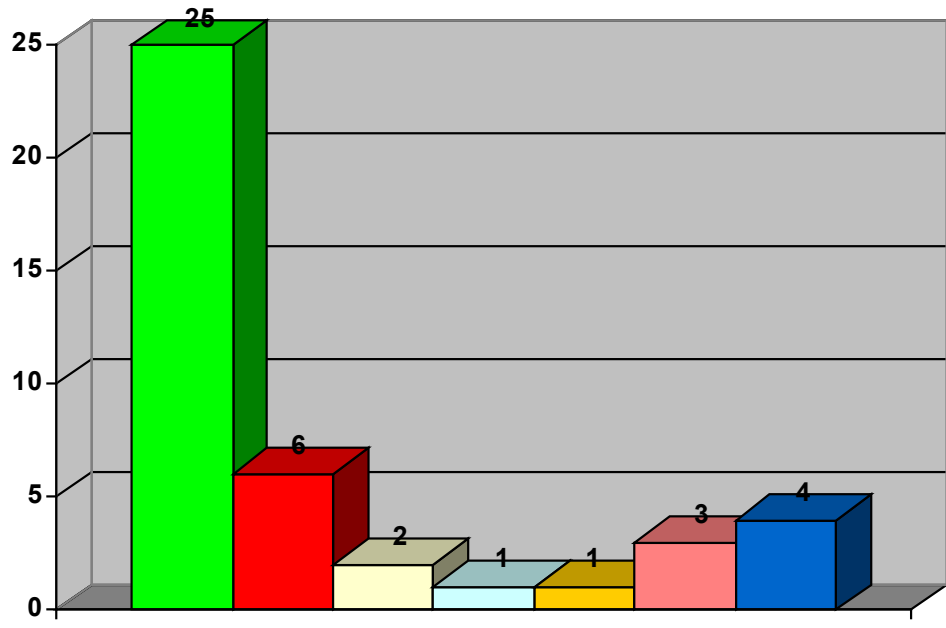

\begin{tabular}{|l|}
\hline Biliar \\
$\square$ Alcohol \\
$\square$ Postquirúrgica \\
$\square$ Fármacos \\
$\square$ Metabólica \\
$\square$ Tumoral \\
$\square$ Idiopática \\
\hline
\end{tabular}

Figura 1. Etiología de la pancreatitis aguda. (En cada barra se expresa el número absoluto de pacientes para cada etiología).

\section{DISCUSIÓN}

La etiología más frecuente de pancreatitis aguda en nuestra serie es la biliar seguida del alcohol. La determinación de tripsinógeno urinario tuvo una positividad en torno al $81 \%$, muy similar a la de la amilasa plasmática que fue del $78 \%$ y ligeramente inferior a la de la lipasa que fue del $85 \%$. En nuestro estudio no se evidenció asociación entre niveles altos de tripsinógeno-2 urinario y mayor estancia media, mortalidad o necesidad de traslado a UCI si bien esto puede ser debido a un sesgo por tratarse de un número no muy elevado de pacientes.

La determinación del tripsinógeno-2 urinario o forma aniónica isoenzimática del tripsinógeno ${ }^{8}$ se ha postulado como un método rápido y sencillo para disminuir el número de diagnósticos erróneos de PA en pacientes que acuden a un servicio de urgencias, en atención primaria u otro tipo de consultas que carecen del acceso rápido a un laboratorio de bioquímica ${ }^{9-13}$ y en pacientes que desarrollan una PA tras ser sometidos a una ERCP ${ }^{14,15}$. En la PA, la elevación del tripsinógeno-2 en orina se evidencia a las pocas horas del comienzo de la PA y desaparece a los tres días ${ }^{16-18}$. En nuestro estudio esta determinación fue positiva en el $81 \%$ de los pacientes con PA, siendo ligeramente superior a lo descrito por Sáez y col $(68 \%)^{19}$ o Lempinen y col $(62 \%)^{20}$ e inferior, pero también equiparable, a lo des- 
crito en otras series que oscila en torno al 85\%-95\% ${ }^{9-13}$. Esto puede explicarse en parte por el hecho de que todos los pacientes incluidos en el estudio tenían el diagnóstico de PA mientras que, al menos en el de Sáez y col, se incluyeron pacientes cuyo diagnóstico final no fue el de PA.

La amilasa sérica debe elevarse al menos tres veces su valor normal aceptándose un punto de corte de $300 \mathrm{U} / \mathrm{L}$ como referencia de positividad pero un $19 \%$ de casos de PA tienen amilasemia normal ${ }^{21}$. Sus niveles pueden elevarse en pacientes con dolor abdominal no pancreático ${ }^{6,22}$.

La lipasa se mantiene más tiempo elevada que la amilasemia o el tripsinógeno-2 urinario por lo que es muy útil en casos de presentación tórpida, pero no es específica del páncreas, pudiendo elevarse en insuficiencia renal crónica u otras patologías intraabdominales. Para algunos autores es preferible a la determinación de amilasa en el diagnóstico de $\mathrm{PA}^{23}$. En nuestro estudio se incluyeron pacientes con amilasa y/o lipasa elevadas para tratar de incluir a todos los pacientes con muy alta sospecha de PA en el momento del ingreso. Se ha descrito que el tripsinógeno-2 urinario tiene una sensibilidad del $94 \%$ y una especificidad del 95\% para el diagnóstico de PA, superior a lo descrito para la amilasa plasmática (sensibilidad del $85 \%$ y especificidad del $91 \%$ ) y para la lipasa (sensibilidad del $79 \%$ y especificidad del $88 \%)^{7,9}$. En nuestro estudio la sensibilidad fue ligeramente inferior y no se analizó la especificidad al carecer de grupo control ni tampoco incluir otros pacientes con dolor abdominal diferente del causado por una PA al no haberse diseñado el estudio para tal fin. Sin embargo, el alto porcentaje de pacientes con tripsinógeno-2 positivo, superior a la amilasemia e inferior a la lipasa, indica que la capacidad de este test para apoyar el diagnóstico de PA es al menos tan buena como las otras dos determinaciones. Esta prueba también tiene una serie de falsos positivos como las neoplasias pancreáticas, diabetes mellitus, hipercalcemia, hipertrigliceridemia, insuficiencia renal crónica, cirrosis hepática, pancreatitis crónica e ictericia obstructiva extrahepática ${ }^{6,24}$.
En nuestro estudio, la presencia de un test de tripsinógeno-2 urinario positivo se asoció de forma clara con una mayor edad en el momento del diagnóstico, elevación de amilasa y lipasa e hipoxemia. La asociación entre amilasemia, lipasa y tripsinógeno-2 urinario se ha descrito en estudios previos en los que se ha visto que el tripsinógeno-2 urinario tiene una sensibilidad y una especificidad superior a la amilasa plasmática con un valor predictivo negativo del $99 \%{ }^{25}$. Resultados similares han sido publicados con respecto a la lipasa plasmática ${ }^{13}$, con un valor predictivo negativo también del 99\% para el tripsinógeno-2 urinario. En estos dos estudios previos la sensibilidad y especificidad para el tripsinógeno-2 urinario en el diagnóstico de PA fue del $94 \%$ y $95 \%$ respectivamente con respecto a la amilasemia y del $93 \%$ y $92 \%$ respectivamente en relación a la lipasa con un punto de corte de $50 \mu \mathrm{g} / \mathrm{L}$. Si la dilución se aumenta a $2.000 \mu \mathrm{g} / \mathrm{L}$, la especificidad se mantiene en el $87 \%$ pero la sensibilidad cae al $62 \%$. Para otros autores, la sensibilidad de la amilasa y la lipasa plasmáticas es mayor que la del trisinógeno-2 urinario (74\%, $84 \%$ y $68 \%$ respectivamente), con una especificidad muy similar para los tres test en torno al $85 \%^{19}$. Las sensibilidades en nuestra serie son similares a las descritas en este último trabajo, también español, y no tan altas como en los trabajos previos finlandeses por lo que no podemos excluir un factor poblacional.

La hipoxemia se ha relacionado con la aparición de complicaciones pulmonares ${ }^{26}$. En nuestro estudio, la asociación entre hipoxemia y positividad para el tripsinógeno-2 urinario iría en consonancia con lo anteriormente explicado; sin embargo, el análisis de las complicaciones pleuropulmonares asociadas no ha sido objeto del presente trabajo.

En cuanto a la estancia media, ésta fue de 8,38 días, siendo en $9(21,4 \%)$ pacientes mayor de 10 días. Este incremento se asoció con una edad superior a 65 años y con la presencia de acidosis metabólica. Esta relación se mantuvo, ajustando por edad, en el análisis de regresión lineal múltiple 
para la estancia media, no así para la acidosis metabólica. La edad es un importante marcador de estancia hospitalaria prolongada por motivos derivados de la propia PA y por la mayor comorbilidad asociada. La acidosis metabólica se ha postulado como una de las causas del dolor en los pacientes con pancreatitis ${ }^{27}$. En este sentido, una mayor acidosis conllevaría mayor dolor y la dificultad en controlar éste último, ligado al grado de inflamación pancreática implicaría un aumento en la estancia del paciente en el hospital. Sin embargo, tras el análisis de regresión lineal múltiple sólo la estancia media mostró asociación cuando se ajustó por la edad y no así la acidosis metabólica ni las otras variables.

Con respecto a la mortalidad, un total de $3(7,14 \%)$ pacientes fallecieron. Dos de ellos habían sido trasladados a una UCI. El tercero presentaba edad avanzada y patología oncológica asociada de base. La mortalidad se asoció con la presencia de hipocalcemia y acidosis metabólica. En pacientes con necrosis pancreática con o sin sepsis asociada, la hipocalcemia ha sido descrita al igual que la insuficiencia respiratoria y la acidosis metabólica como factor de mal pronóstico en pacientes con $\mathrm{PA}^{28,29}$. Se ha descrito que niveles elevados de tripsinógeno-2 urinario se asocian a un incremento en la mortalidad por $\mathrm{PA}^{30}$, sin embargo, en nuestro estudio no podemos llegar a esta conclusión probablemente porque el bajo número de pacientes fallecidos no hace factible la obtención de conclusiones fiables. A pesar de ello, en 2 de los pacientes fallecidos, la determinación de tripsinógeno-2 urinario fue superior a $2.000 \mu \mathrm{g} / \mathrm{L}$. Probablemente la acidosis metabólica, hipocalcemia e hipoxemia representen un papel importante en la génesis de la gravedad de la PA contribuyendo a un aumento de la estancia hospitalaria y de la mortalidad y morbilidad asociadas.

Concluyendo, la determinación del tripsinógeno-2 urinario en pacientes con PA es un test útil y rápido equiparable a la determinación de amilasa o lipasa plasmáticas para el diagnóstico de esta patología. En nuestro estudio, los pacientes con ma- yor edad muestran un resultado positivo con más frecuencia que los menores de 65 años. Esta determinación de tripsinógeno-2 urinario positiva, junto con la presencia de hipoxemia y acidosis metabólica en la gasometría arterial e hipocalcemia, puede ser de utilidad en la valoración pronóstica de los pacientes ingresados por PA con respecto a la probabilidad de presentar un incremento en su la estancia media en el hospital y una mayor probabilidad de fallecimiento por esta enfermedad en el curso del ingreso hospitalario.

\section{BIBLIOGRAFÍA}

1. Goldacre MJ, Roberts SE. Hospital admission for acute pancreatitis in an English population, 1963-98: database study of incidence and mortality. BMJ 2004; 328: 1466-1469.

2. Swaroop VS, Chari ST, Clain JE. Severe acute pancreatitis. JAMA 2004; 291: 2865-2868.

3. Toh SK, Phillips S, Johnson CD. A prospective audit against national standards of the presentation and management of acute pancreatitis in the South of England. Gut 2000; 46: 239-243.

4. Rettally CA, Skarda S, Garza MA, Schenker S. The usefulness of laboratory tests in the early assessment of severity of acute pancreatitis. Crit Rev Clin Lab Sci 2003; 40: 117149.

5. Borda F, Oquiñena S. Borobio E, Vila J, Frauca C, Martínez B. ¿Tiene utilidad el tratamiento preoperatorio con ácido ursodeoxicólico en la reducción de las recidivas en la pancreatitis aguda biliar? An Sist Sanit Navar 2003; 26 : 225-229.

6. Yadav D, Agarwal N, Pitchumoni CS. A critical evaluation of laboratory tests in acute pancreatitis. Am J Gastroenterol 2002; 97: 13091318.

7. KylänpäÄ-Bäck ML, Kemppainen E, PuolakKainen P, Hedström J, Haapiainen R, Korvuo A et al. Comparison of urine trypsinogen-2 test strip with serum lipase in the diagnosis of acute pancreatitis. Hepatogastroenterology 2002; 49: 1130-1134.

8. Petersson U, Appelros S, Borgström A. Different patterns in immunoreactive anionic and cationic trypsinogen in urine and serum in human acute pancreatitis. Int J Pancreatol 1999; 25: 165-170. 
9. Kemppainen EA, Hedström Ji, Puolakkainen PA, Sainio VS, HaApiainen RK, Perhoniemi V et al. Rapid measurement of urinary trypsinogen-2 as a screening test for acute pancreatitis. N Engl J Med 1997; 336: 1788-1193.

10. Hedström J, Korvuo A, Kenkimäki P, Tikanoja S, HaAPIAINEN R, KivilaAKSo E et al. Urinary trypsinogen-2 test strip for acute pancreatitis. Lancet 1996; 347: 729-730.

11. Pezzilli R, Morselli-Labate AM, d`Alessandro A, BARAKAT B. Time-course and clinical value of the urine trypsinogen-2 dipstick test in acute pancreatitis. Eur J Gastroenterol Hepatol 2001; 13: 269-274.

12. Kemprainen E, Hedström J, Puolakkainen P, HalttUNEn J, SAinio V, HaApiainen $\mathrm{R}$ et al. Urinary trypsinogen-2 test strip in detecting ERCPinduced pancreatitis. Endoscopy 1997; 29: 247-251.

13. KylänpäÄ-Bäck M, Kemppainen E, PuolakKainen P, Hedström J, Haapiainen R, Perhoniemi V et al. Reliable screening for acute pancreatitis with rapid urine trypsinogen-2 test strip. Br J Surg 2000; 87: 49-52.

14. Sankaralingam S, Wesen C, Barawi M, Galera R, LLOYD L. Use of the urinary trypsinogen-2 dip stick test in early diagnosis of pancreatitis after endoscopic retrograde cholangiopancreatography. Surg Endosc 2007; 21: 1312-1315.

15. BAILlE J. Predicting and preventing postERCP pancreatitis. Curr Gastroenterol Rep 2002; 4: 112-119.

16. Borgström A, Appelros S. Activation peptides in acute pancreatitis. En: Büchler MW, Uhl W, Friess H, Malfertheiner P, ed. Acute pancreatitis. Novel concepts in biology and therapy. Blackwell Wissenschafts-Verlag. Berlin-Viena 1999; 219-223.

17. Matull WR, Pereira SP, O`Donohue JW. Biochemical markers of acute pancreatitis. J Clin Pathol 2006; 59: 340-344.

18. Kemppainen E, Hedström J, Puolakkainen P, HalttUnen J, Sainio V, HaApiainen R et al. Increased serum trypsinogen 2 and trypsin 2-alpha 1 antitrypsin complex values identify endoscopic retrograde cholangiopancreatography induced pancreatitis with high accuracy. Gut 1997; 41: 690-695.

19. Sáez J, Martínez J, Trigo C, Sánchez-Payá J, ComPAÑY L, LAVEDA R et al. Clinical value of rapid urine trypsinogen-2 test strip, urinary trypsinogen activation peptide, and serum and urinary activation peptide of carboxypeptidase
$\mathrm{B}$ in acute pancreatitis. World $\mathrm{J}$ Gastroenterol 2005; 11: 7261-7265.

20. Lempinen M, KylänpäÄ-Bäck ML, Stenman UH, Puolakkainen P, HaApiainen R, Finne $\mathrm{P}$ et al. Predicting the severity of acute pancreatitis by rapid measurement of trypsinogen-2 in urine. Clin Chem 2001; 47: 2103-2107.

21. Clavien PA, Robert J, Meyer P, Borst F, Hauser $\mathrm{H}$, Herrmann $\mathrm{F}$ et al. Acute pancreatitis and normoamylasemia. Not an uncommon combination. Ann Surg 1989; 210: 614-620.

22. Terada T, NaKAnuma Y. Immunohistochemical demonstration of pancreatic alpha-amylase and trypsin in intrahepatic bile ducts and peribiliary glands. Hepatology 1991; 14: 1129-1135.

23. Werner J, Feuerbach S, Uhl W, Büchler MW. Management of acute pancreatitis: from surgery to interventional intensive care. Gut 2005; 54: 426-436.

24. Malvano R, Marchisio M, Massaglia A, Giacosa PA, Zannino M, Andriulli A et al. Radioimmunoassay of trypsin-like substance in human serum. Scand J Gastroenterol 1980; 62: 3-10.

25. Kemppainen EA, Hedstrom J, Puolakkainen P, SaInio V, Haapiainen R, Perhoniemi V et al. Rapid measurement of urinary trypsinogen- 2 as a screening test for acute pancreatitis. N Engl J Med 1997; 336: 1788-1793.

26. Martín Alonso MA, Santamaría A, Saracíbar E, Arranz E, Garrote JA, Almaraz A et al. Citocinas y otros parámetros inmunológicos como marcadores de alteración de órganos a distancia en la pancreatitis aguda. Med Clin (Barc) 2007; 128: 401-406.

27. Bornman PC, Marks IN, Girdwood AW, Berberat PO, Gulbinas A, Büchler MW. Pathogenesis of pain in chronic pancreatitis: ongoing enigma. World J Surg 2003; 27: 1175-1182.

28. Desai TK, Carlson RW, Geheb MA. Prevalence and clinical implications of hypocalcemia in acutely ill patients in a medical intensive care setting. Am J Med 1988; 84: 209-214.

29. Bittner R, Block S, Büchler M, Beger HG. Pancreatic abscess and infected pancreatic necrosis. Different local septic complications in acute pancreatitis. Dig Dis Sci 1987; 32: 1082-1087.

30. Pitchumoni CS, Patel NM, Shah P. Factors influencing mortality in acute pancreatitis: can we alter them? J Clin Gastroenterol 2005; 39 : 798-814. 